\title{
Cloning of High Molecular Weight Gluten Subunit Promoter and Study on its Function in Wheat
}

\author{
Jin Weibo ${ }^{1,2}$, Liu Jin ${ }^{1,2}$, Wu Fangli ${ }^{1}$ and Guo Aiguang ${ }^{1,2^{*}}$ \\ ${ }^{I}$ College of Life Science; Northwest A\&F University Yangling; 712100; Shaanxi - China. ${ }^{2}$ Key Laboratory of \\ Agriculture Molecular Biology in Shannxi; Yangling; 712100; Shaanxi - China
}

\begin{abstract}
The aim of this work was to study the cloning and characterization of HMW-GS 1Dx2 promoter from Triticum aestivum. A $1050 \mathrm{bp}$ partial promoter fragment including a putative TATA box and 5' encoding sequence of the gene was cloned by amplifying the upstream sequences using the nest-PCR with appropriate primers. The analysis of the promoter sequence against the PLACE (Plant cis-acting Regulatory DNA Elements) database showed the presence of certain putative endosperm-specific regulatory cis-elements in the sequence along with the TATA and CAAT boxes. The histochemical method detected the transient expressions of GUS in the seeds of wheat. The results showed that HMW-GS 1Dx2 promoter had the endosperm-specific transcription activity in the wheat seeds.
\end{abstract}

Key words: Triticum aestivum; glutenin; promoter; endosperm-specific; transient expression

\section{INTRODUCTION}

Cereal grains provide a significant proportion of human proteins and calories intake. The use of the wheat flour in the production of leavened bread products is dependant on the seed storage proteins (SSP) that accumulate in a developing wheat grain because they play a pivotal role in the visco/elastic properties of dough. Unlike other cereals, only wheat has a suitable combination of the seed storage proteins to produce the leavened bread products(Shewry et al., 1994). The genetic transformation provides a means of introducing the novel genes for modifying the cereal grain characteristics. For example, the transgenes can be used to alter the relative amounts of the specific proteins or starch components to make new or improved processed products. For this purpose, it is essential to clone the promoters that drive high levels of the transgene expression in the endosperm. Wheat $H M W-G S$ gene promoters are, in principle, ideal candidates to drive the transgene expression in the cereal endosperm, as high levels of the prolamin synthesis occur (cell expansion) during the linear phase of the seed development(Forde et al., 1985; Sugiyama et al., 1985; Halford et al., 1987; Carmela et al., 2001).

\section{MATERIALS AND METHODS}

\section{Materials}

The seeds of Xiaoyan 6 were germinated and grown for 10-12 days without light in the laboratory. The genomic DNA was isolated from the young leaves of Xiaoyan 6 by CTAB method(Rogers \& Bendich, 1994).

\footnotetext{
* Author for correspondence: guoaiguang@yahoo.com.cn
} 


\section{Amplification of $H M W-G S$ gene promoter} The homologous primers of the $H M W-G S$ gene promoter for PCR on the genomic DNA was used. Then a secondary PCR was done for cloning the $H M W-G S 1 D \times 2$ promoter with the anchor primers. The anchor primers used in the reactions were Sense ( $5^{\prime}>$ GGAAGCTTAGTGATGGCGTGAG $<3$ ') and Anti-Sense primer (5'> TCGGATCCCTCACCTTCAGCG $<3$ '). After each PCR, $10 \mu \mathrm{l}$ of the PCR products was checked in a $1.5 \%$ agarose gel. After the secondary PCR, the relevant amplification products were eluted from the gel using the PCR DNA and gel band purification kit (Sangon) and cloned into pMD18$\mathrm{T}$ vector(Takara). The inserts were sequenced by Sangon.

\section{Sequence analysis of the cloned fragment}

The promoter sequence was analyzed by the PLACE program (http://www.dna.affrc.go.jp/htdocs/PLACE/) for the theoretical identification of the regulatory element binding sites against PLACE database(Higo et al., 1999). Moreover, the evolution analysis of the sequence obtained was carried out for its chromosomal location with the DNAstrar program.

\section{Wheat transformation}

The promoter-reporter construct(pMHG), the positive control pBI121 and the negative control pMG were transformed into wheat leaves and immature seeds via the particle bombardment following the standard protocols $(\mathrm{Xu} \&$ Wei, 1998). The expression of uidA gene under the influence of different promoter constructs was analysed by the fluorometric assay(Jefferson et al., 1997).

\section{RESULTS AND DISCUSSIONS}

\section{Amplification of HMW-GS gene promoter} In this study, the nest PCR approach was followed to clone the $H M W-G S$ promoter from the genome DNA. After the second round of the amplification, the approximately $1 \mathrm{~kb}$ long products were obtained (Fig. 1).

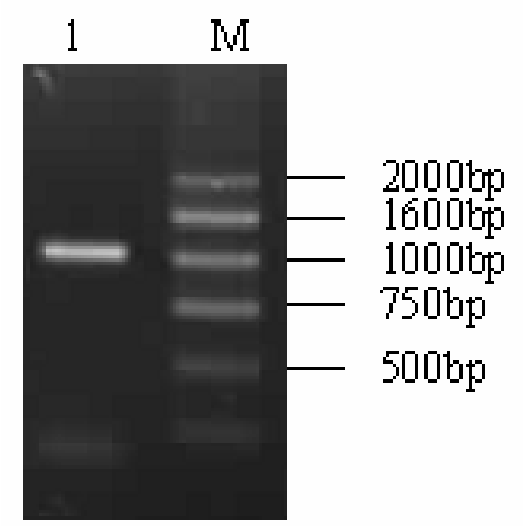

Figure 1 - Agarose gel electrophoresis after secondary PCR. Lane M: molecular marker DGL2000; Lane 1: PCR products (about 1K).

\section{Motifs analysis of the cloned fragment}

Online BLAST analysis and ClustalW multiple alignment indicated that there were many sequences similarity of this sequence with other known HMW-GS promoter sequences.

The sequence was analyzed for the presence of the plant cis-acting regulatory DNA elements through the PLACE signal scanning. In Fig. 2, the presence of a TATA box was identified $91 \mathrm{bp}$ upstream to the translation start site of the $H M W-G S$. Other important motifs found included the CAAT box, A , $\mathrm{B}$ and $\mathrm{C}$ consensus sequence and $\mathrm{CAC}$ box along with other putative transcription factor binding sites. The TATA box and CAAT box were upstream sequence elements responsible for the proper transcription event. A, B and C motifs were conserved sequences concerning the cereal storing protein expression (Huang et al., 1995). Both A and $\mathrm{C}$ were conservative elements in the homogeneous sequence of the published wheat 
HMW-GS 5' upstream. Furthermore, they have middle conservation with $5^{\prime}$ upstream different region of several kinds $\alpha / \beta$ type gliadin gene, and were predicted that they have relation to gene expression and regulation. B element was "-300 element", whose central sequence were TGCAAAA or TCTAAAG. It existed in the 5, conserved region of many cereal storing protein genes, including the HMW-GS and played a significant role in the endosperm-specific expression. Based on these results, it could be hypothesized that the $H M W-G S$ gene promoter might be a endosperm-specific promoter.

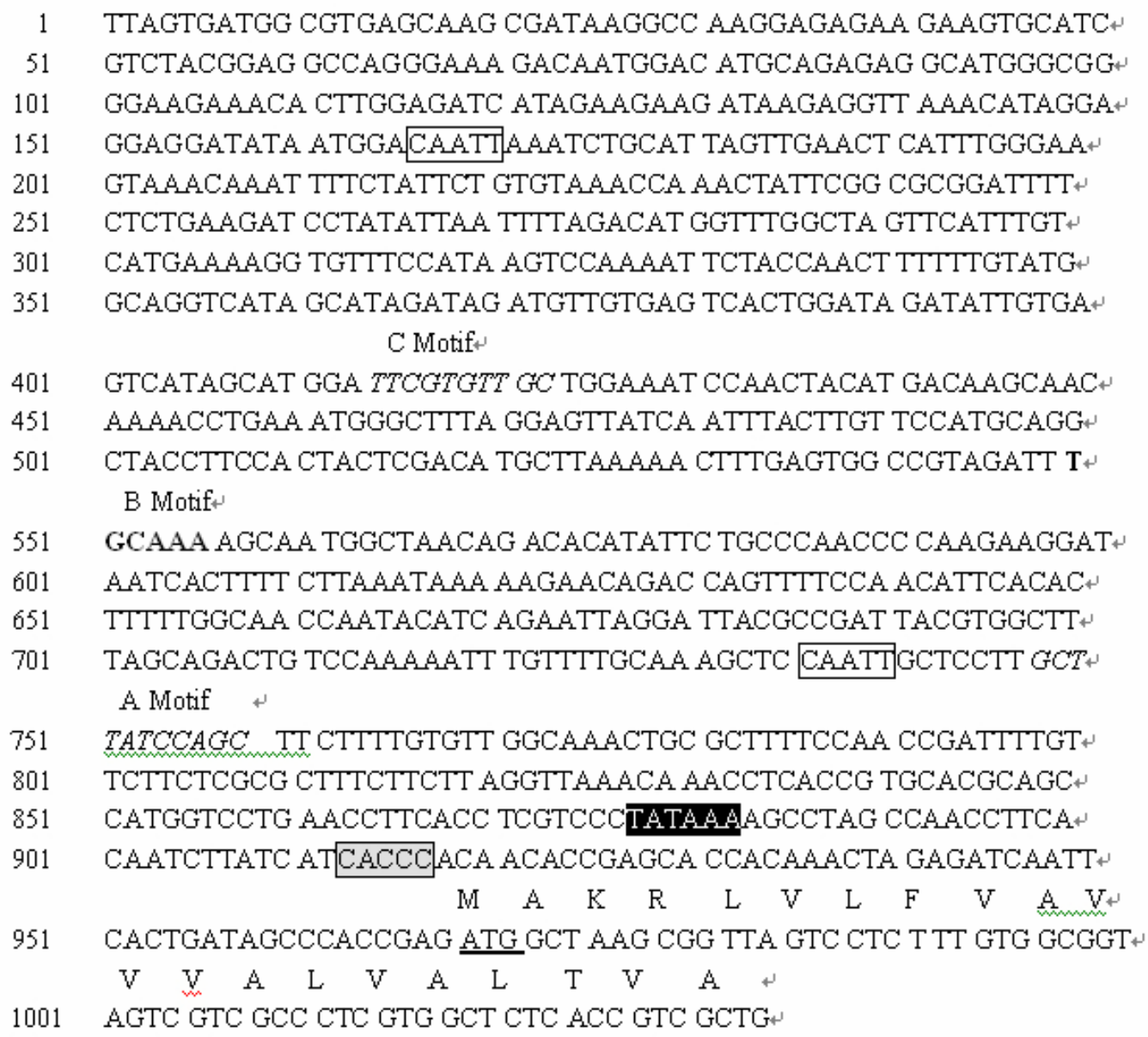

Figure 2 - The cloned promoter sequence of HMW-GS. The 5' upstream region along with first tentyone amino acids of signal-peptide is given. The translation initiation codon ATG is underlined. Putative TATA box and CAAT box, -300 elements core, CAC box etc. are identified by analyzing the sequence by PLACE algorithm. The black box shows the TATA box motif. The gray box region represents the CAC motif. The white boxs shows the CAAT box motif. The bold nucleotides shows the "-300 elements" and italic nucleotides regions represent Motif core of the seed-specific expression cis-elements. The cloned promoter sequence data can be obtained from EMBL under the accession number AJ577815. 


\section{Chromosome assignment of the promoter}

To know that the promoter belonged to which HMW-GS, the phylogenetic analysis of 43 homologous fragments of $H M W-G S$ was conducted using the 5' flanking sequences and the sequences encoding the signal peptides. In Fig.3, the cloned fragment was underlined. It showed that the cloned $1 \mathrm{~kb}$ fragment was type $\mathrm{X} H M W-G S$ gene promoter on 1D chromosome. Since $H M W$ $G S$ on $1 \mathrm{D}$ chromosome in the wheat Xiaoyan 6 was subunit 2 , a conclusion could be drawn that the cloned fragment consisted of wheat $H M W-G S$ $1 D \times 2$ promoter and signal peptide region.

\section{Vector construction of transient expression}

The promoter fragment from the pMD18-T vector was fusion with the GUS gene from pBI121 to get the pMHG (Fig.4). Furthermore, for the promoter analysis, the fragment of GUS gene without promoter was cloned into the pMD18-T vector named pMG as the negative control and pBI121 with uidA gene downstream of CaMV $35 \mathrm{~S}$ promoter as the positive control.

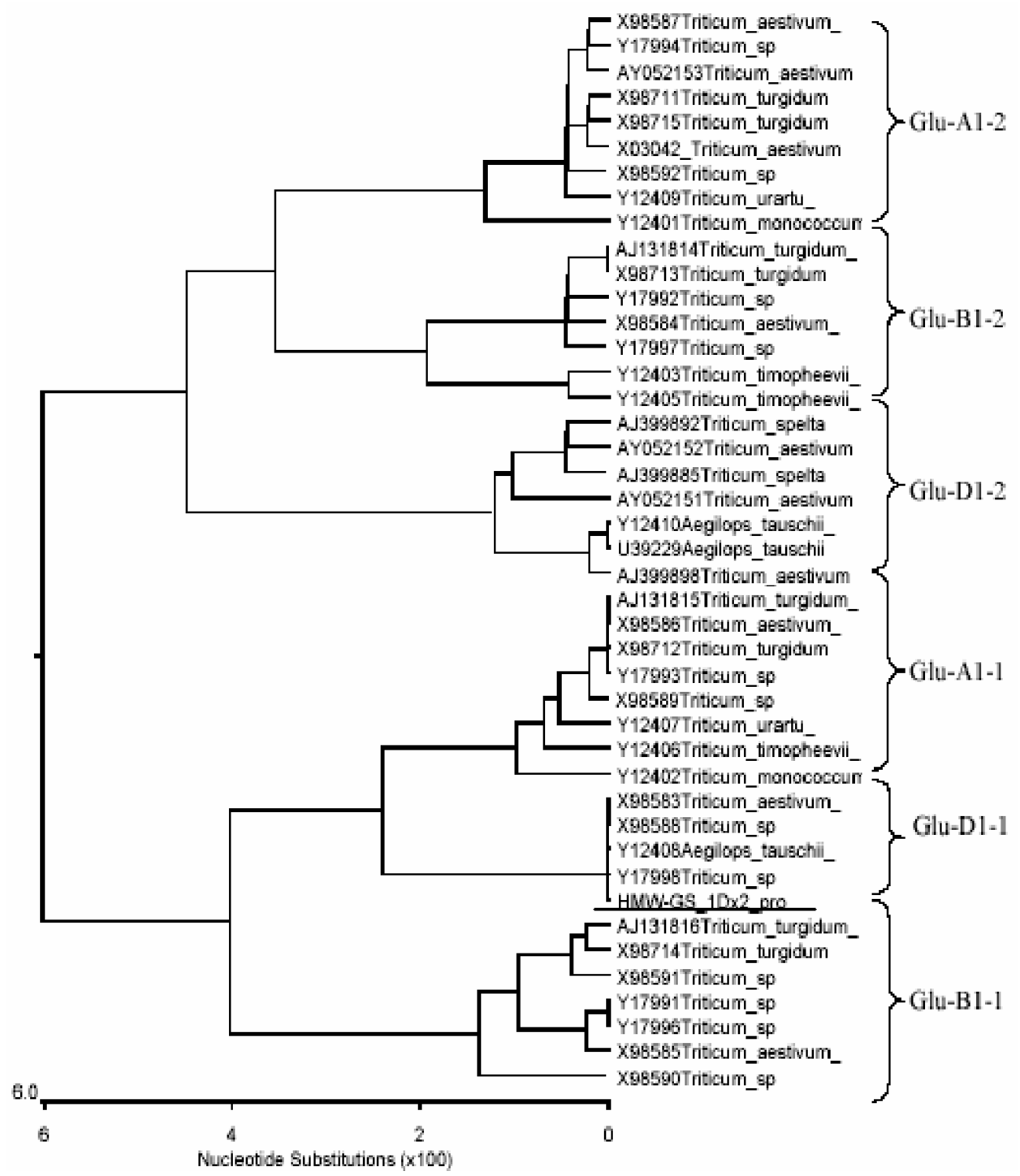

Figure 3 - Phylogenetic relationship of the cloned fragment(underline) with previously publicized $H M W-G S$ promoters. The rootless phylogenetic tree was constructed based on a multiple alignment of the 5' flanking sequences and the sequences encoding the signal peptides of the $42 H M W-G S$. 


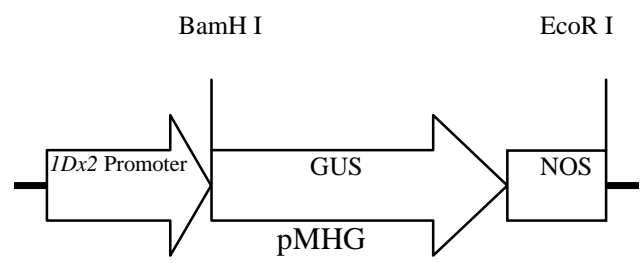

Figure 4 - Scheme for construction of plasmid pMHG. Step I: Cloning PCR product and Isolate fragment of GUS+NOS with BamHI and EcoRI; StepII: Insertion of Gus gene after HMW-GS promoter.

\section{Function determination of wheat $H M W-G S$ promoter}

The reporter gene expression from an introduced promoter could be accomplished by either the transcriptional or translational fusions. The golden powder was coated with PBI 121, pMHG and pMG, and then transformed the wheat leaves and seeds via the particle bombardment. The GUS activity was examined by the fluorometric assay. In Fig.5, the GUS activity were very weak in the negative control(pMG), which was possibly caused by the system error or intrinsic fluorescence, but had no effect on the experiment result. The transformed seeds and leaves with pBI 121 , the positive control could be identified with the GUS expression, although it was at a lower activity. However, the seeds and leaves transformed with the pMHG, the activity turned out about 2.5 times as high as pBI121 in the seeds but for the leaves it was lower. Based on these results it could be inferred that the HMW-GS $1 D x 2$ promoter had tissue-specific activity in the wheat seeds.

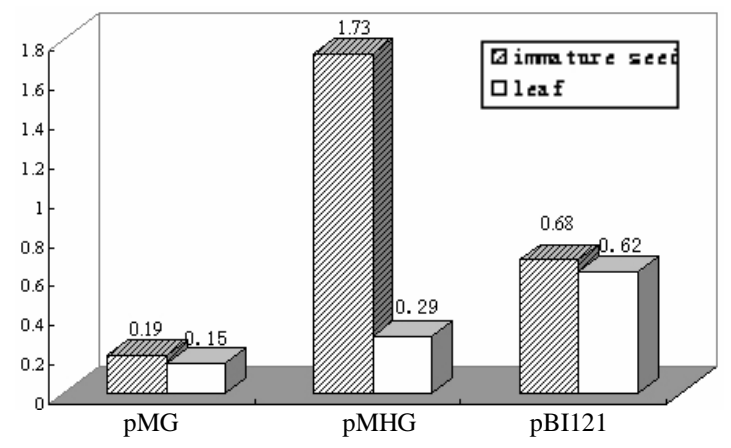

Figure 5 - Transient expression assay for wheat endosperm-specific HMW-GS gene promoter; pMG: no promoter, negative control; pMHG: Promoter of wheat endosperm-specific HMW-GS gene; pBI121: CaMV 35S promoter, positive control.

\section{CONCLUSION}

It was concluded that the promoter region of T.aestivum $H M W$-GS was cloned, which showed the presence of endosperm-specific regulatory cisacting elements. The 1050 bp promoter sequence including TATA box and downstream signal peptide were expressed at high level for the GUS gene than the CaMV 35S promoter in the seeds of the wheat. The DNA sequence has been submitted into EMBL Nucleotide Sequence Database. The accession number is AJ577815. 


\section{ACKNOWLEDGMENTS}

This work was supported by the Department of Science and Technology in China (JY03A-11-01) and $\mathrm{Ph} . \mathrm{D}$ innovation foundation.

\section{REFERENCES}

Carmela, L, Peter, R.S, Natale, D.F, Jane, L.F, Nick, H, Paul, A.L, Johnathan, A.N, Nigel, G.H, Pilar, B. (2001), Endosperm-specific activity of a storage protein gene promoter in transgenic wheat seed. $J$. Exp. Bot., 52, 243-250.

Forde, J, Malpica, J.M, Halford, N.G, Shewry, P.R, Anderson, O.D, Greene, F.C, Miflin, B.J, Anderson, O.D, Greene, F.C and Miflin, B.J. (1985), The nucleotide sequence of a HMW glutenin subunit gene located on chromosome 1A of wheat(Triticum aestivum L.). Nucl. Acids Res, 13, 6817-6832.

Halford, N.G, Forde, J, Anderson, O.D, Greene,F.C and Shewry, P.R. (1987), The nucleotide and deduced amino acid sequences of an HMW glutenin subunit gene from chromosome $1 \mathrm{~B}$ of bread wheat (Triticum aesfivum L.) and comparison with those of genes from chromosomes 1A and 1D. Theor. Appl. Genet, 75, 117-126.

Higo, K, Ugawa, Y, Iwamoto, $M$ and Korenaga, T. (1999), Plant cis-acting regulatory DNA elements (PLACE) database. Nucleic Acids Res, 27, 297-300
Huang, W, Jiang X, Huang, H, He, B and Sun, C. (1995), Preliminary Study on cis-trans interaction in transcriptional regulation of a wheat high-molecularweight(HMW) glutenin gene. Acta Botanica Sinica, 37, 425-431.

Jefferson, R.A, Kavanagh, T.A, Bevan, M.W. (1987), GUS fusions: beta-glucuronidase as a sensitive and versatile gene fusion marker in higher plants. EMBO J., 6, 3901-3907.

Rogers, S.O and Bendich, A.J. (1994), In-Plant molecular biology manual. 2nd Edn, ed. S.B. Gelvin, R.A. Schilperoort. Kluwer, pp. D1:1-8.

Shewry, P.R, Miles, M.J and Tatham, A.S. (1994), The prolamin storage proteins of wheat and related cereals. Prog. Biophys.Mol. Biol, 61, 37-59.

Sugiyama, T, Rafalksi, A, Peterson, D and Soll, D. (1985), A wheat HMW glutenin subunit gene reveals a highly repeated structure. Nucl. Acids Res, 13, 8729-8737.

$\mathrm{Xu}, \mathrm{S}$ and Wei, Z. (1998), Introduction to Method of Microprojectile Bombardment and Its Application. Plant Physiology Communication, 34, 41-45.

Received: June 12, 2006; Revised: April 10, 2007; Accepted: June 04, 2008. 